\title{
DESIGN AND RESEARCH OF HARDWARE CIRCUIT BASED ON INTELLIGENT AIRBORNE VOICE INFORMATION SYSTEM
}

\author{
Honglei Shen \\ Suzhou Industrial Park Industrial Technology School, Jiangsu Lianhe Technical Institute, \\ Jiangsu, China \\ E-mail: shenhongleishl@163.com
}

\begin{abstract}
In order to develop a new hardware circuit equipped with voice information system, the hardware circuit based on intelligent airborne voice information system is designed. In different stages of the flight, the voice information system can provide information about flight status, flight status and the required operation for the flight crew and the ground command personnel. At the same time, it can also carry out voice alarm, so the pilot can concentrate on manipulating the aircraft. The role of the voice information system is not to be questioned. It is one of the most important equipment in the airborne radio equipment. Based on this, the hardware circuit of the Intelligent Airborne voice information system is designed. According to the circuit characteristics of the system, from the point of view of convenient maintenance, the whole circuit is divided into four parts: input unit, control unit, audio unit and output unit. Through this design, the dependence of accessories on imported products is solved, and the cost is reduced. In maintenance, manpower, material resources and time are relatively reduced. The experimental results show that the improved system fundamentally overcome the defects of the original system of steel tape broadcast mode. Meanwhile, it realizes the intelligence, solid and integration of the airborne voice information system.
\end{abstract}

Keywords: Intelligent Airborne; Voice Information System; Audio Unit.

\section{Introduction}

In recent years, our army has imported a large number of large transport planes from abroad. The transport performance of the transporter is outstanding, but its airborne voice information system is a foreign product more than 30 years ago.

Limited by the technical conditions at that time, the selection of relay combination control signal is adopted, and the motion of the magnetic tape is driven by the motor [1-2]. From the protection and maintenance of the transport aircraft in China for nearly ten years, there are many defects in the voice information system, and it is difficult to repair once the fault occurs. In addition, direct delivery of foreign repairs or the purchase of new equipment costs a lot of money. The cycle is long, and it has a direct impact on the completion of the flight mission [3]. With the rapid development of electronic technology in the world today, the application of computer, voice chip and large-scale integrated circuit has brought a revolution to the design of airborne electronic equipment [4-5]. The development direction of airborne avionics is the new integrated system, which is compatible with the new and old systems. It can give full play to all the functions of the new system, and also take into account the use of the old system. It constitutes a secret, highly reliable, multi-functional backup system, and also provides a good way for the transition between the new and the old systems [6].

Modern voice information system can use the advanced electronic technology to develop vigorously along the direction of digital information transmission and intelligent process control [7]. The localization of the voice information system completely abandoned the original system defects.

The application of modern technology and electronic devices fully meets the level of intelligent control. The rapid response capability of aircraft is improved, while the automation, modernization and high reliability requirements of airborne electronic equipment are realized.

\section{The Main Technical Defects of the Original Airborne Voice System}

The original airborne voice information system was a product of foreign countries more than 30 years ago. Restricted by the technical conditions at that time, the motor drives tapes to play voice prompt and warn sound. From more than 10 years of our 
army's support and maintenance, there are many defects in the system. It is mainly manifested in the following aspects [8]: The voice storage medium adopts the steel wire base band tape, and the specifications are special. Therefore, it should be replaced regularly according to the rules of use.

Therefore, the tape is required to keep enough spare parts and have a stable manufacturer or external order. In the process of using, the sound quality of the tape becomes worse, while the distortion and the noise become larger. Long term movement of tapes can easily cause deformation, breakage, winding, mechanical mechanisms and other system failures, which may cause loss of audio signals.

The voice information stored by tapes is affected by mold, moisture, magnetic particle falling off, adhesion and electromagnetic interference. Therefore, based on the widely applied solid state speech processing technology, the domestic intelligent development of transport aircraft voice information system is developed. On the one hand, we can fundamentally overcome the defects of the original system. On the other hand, it can also solve the dependence of foreign material, spare parts and maintenance on the foreign party.

\section{Method}

Intelligent voice information system includes microprocessor, voice storage, voice processor, digital logic information selection component, automatic record and playback conversion circuit, bus communication interface, operation display unit and so on. The block diagram is shown in figure 1.

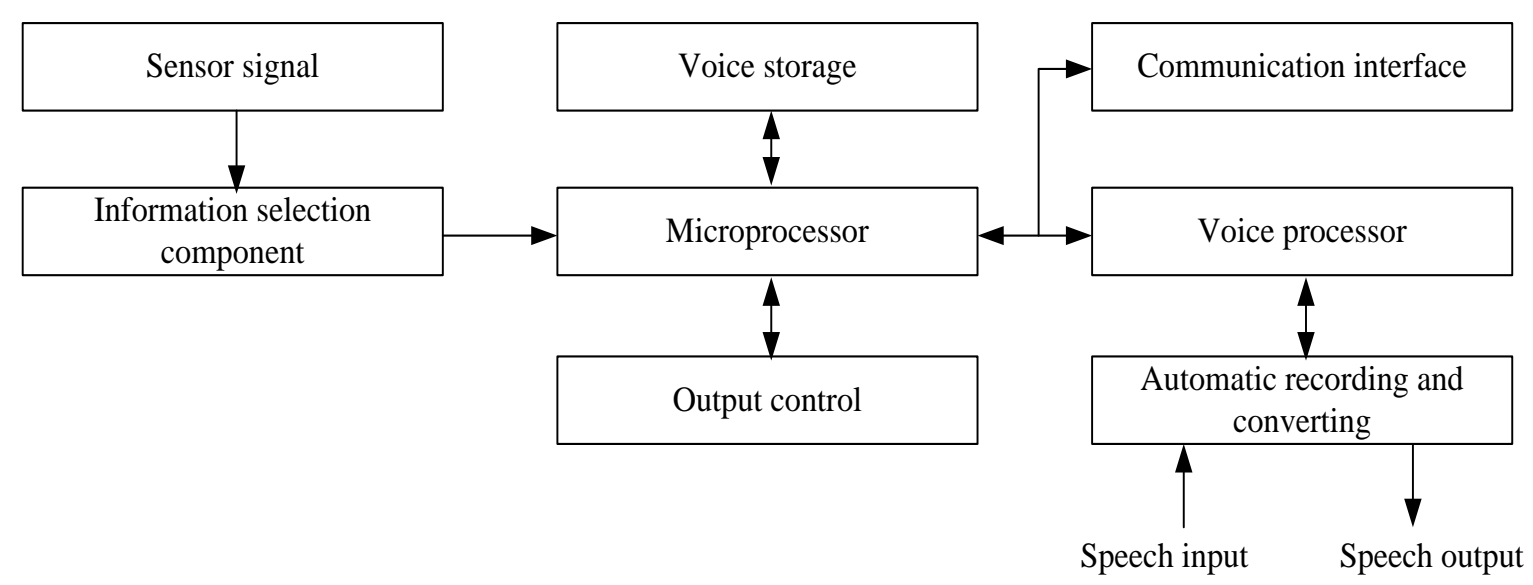

Figure 1 Block diagram of speech information system

The circuit is divided into four units according to the input trigger high voltage signal, digital processing high frequency signal, audio processing lowfrequency signal and output control large current signal. They are designed as four pieces of "page style" structure that can be inserted directly.

According to the characteristics of the signal to be processed, the intelligent voice system is divided into four parts that can be disassembled directly. The four units are input unit, control unit, audio unit and output unit.

\section{Results and Discussion}

The basic working principle of intelligent airborne voice information system: Using the voice storage function of the chip and the peripheral interface circuit, the recording and operation of the voice information can be realized. Different responses can be achieved for different input signals.
That is, each input of a sensor signal can release a corresponding voice information to achieve the function of voice alarm.

\subsection{Input unit}

The input unit corresponds to the information selection component of the original system. The information selection component of the original system consists of 16 sets of identical circuits. Each circuit includes polarization relay, ordinary relay, diode and so on. It mainly depends on the order of closing and opening of different relays to complete the order of importance. In aircraft, there are about more than 200 mechanical sensors with different temperature, voltage, current, smoke, speed, gesture and others. The output signal of the sensor is positive $+27 \mathrm{~V}$ pulse.

The width is greater than $50 \mathrm{~ms}$, and the interval is greater than $50 \mathrm{~ms}$. Its frontier is less than $1 \mathrm{~ms}$, and the slope is steep. The waveform and the circuit are shown in figure 2 . 


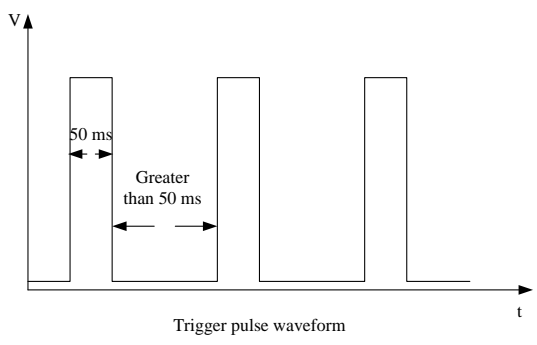

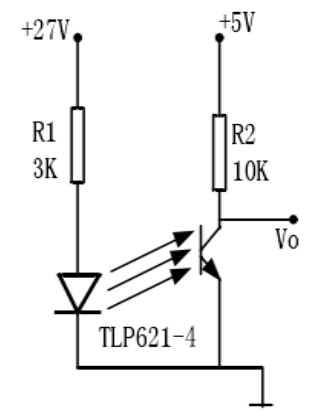

Optocoupler connection circuit

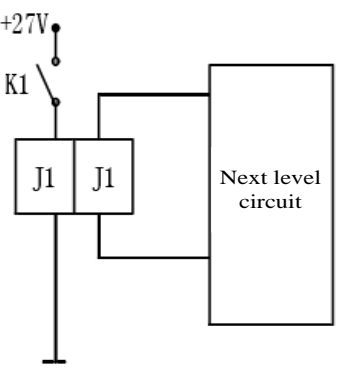

Relay connection circuit

Figure 2 Sensor signal waveform and decomposition of input circuit

The K1 in figure 1 corresponds to the internal switch of the relay J1. Whether the circuit is working or not is decided by the switch of bipolar relay. The sensor has a lower reliability when the signal is switched on.

The input unit of the intelligent voice information system is composed of an optocoupler and an input latch circuit. Here, the TOSHIBA TLP621-4 type optocoupler is used. It is visually connected to a phototransistor by a light emitting diode that emits infrared light. The structure is shown in figure 2

The DIP package of the TLP621-4 optocoupler provides 16 pins and 4 electrically isolated channels. When the +27 -volt analog signal is input, the diode is led to emit light, and the phototransistor is irradiated by light to generate current conduction. When the collector of the phototransistor is connected with a pull resistor, the collector outputs a TTL type low level. When the wireless sensor signal is input, the diode does not emit light and the phototransistor is not connected. The TTL high level is output. Therefore, the photoelectric coupler not only realizes the level conversion, but also plays the role of isolation.

In intelligent systems, when the sensor touches the trigger signal, the diode impedance of the optocoupler is negligible. An electric current of $9 \mathrm{~mA}$ is generated on the diode, and an instantaneous +55 $\mathrm{V}$ induction potential is generated by light irradiation. The phototransistor is turned on. voltage is about $0.2 \mathrm{~V}$. The collector of the phototransistor is connected with a pull-up resistor $\mathrm{R} 2$, and then the collector outputs a TTL type low level. When the wireless sensor signal is input, the phototransistor is only $10 \mathrm{nA}$ when no light is applied. The phototransistor is approximately non conducting and the collector outputs TTL type high level.
The electrical signal output by the optocoupler is delivered to a 16-bit latch consisting of two 54LS373. The cycle is detected by a single chip microcomputer to achieve rapid and accurate playback according to the specified importance and priority sequence. At the same time, the input of other trigger signals during playback is latched and remembered. The wiring diagram of the 54 LS373 is shown in figure 3 .

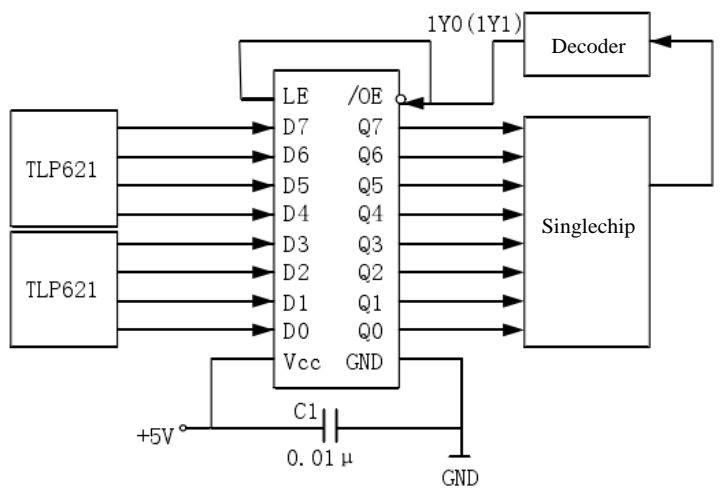

Figure 354 LS373 pin connection diagram

\subsection{Control unit}

The control unit is the core unit of the system, which is equivalent to the tape converter and tape reel mechanism of the original tape system.

The tape converters and tape reel mechanisms of original system can emit sound relying on the right and left rotation of the tape turn by motor. The voice storage medium uses a steel baseband tape. In use, tape quality becomes worse, distortion and noise become larger. Long term movement is easy to deformation, fracture, winding mechanical mechanism, resulting in loss of audio signals, system 
failure. Its failure rate is high and its service life is limited. Eight-channel head needs regular cleaning and replacement. Rotating parts and mechanical contacts should be regularly maintained. The voice information stored by tapes is affected by mold, moisture, magnetic particle falling off, adhesion and electromagnetic interference.

\subsection{Audio unit}

It consists of band-pass filter, power amplifier, output control circuit. It is used to amplify the speech signal under normal broadcast condition and to match the different loads, and to control the output according to the need.

Band-pass filter: Sound signals mix with noise during recording. In order to obtain more pure speech signals, an active band pass filter is set up.
The bandpass filter is characterized by allowing the frequency components in the limited bandwidth to pass smoothly while other frequency components outside this frequency range are attenuated. A lowpass filter is connected with a high pass filter circuit to form a bandpass filter. The condition is that the cut-off angle frequency of the low-pass filter circuit is larger than the cut-off angle frequency of the high pass filter circuit. The block diagram of the bandpass filter is shown in figure 4 .

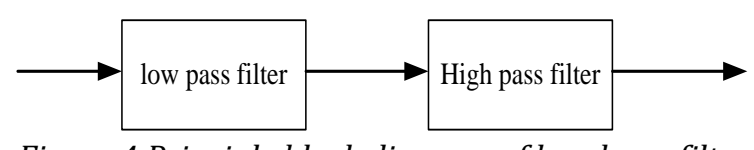

Figure 4 Principle block diagram of bandpass filter

The bandpass filter requires a passband width of 300 to $3400 \mathrm{~Hz}$. The circuit is shown in figure 5 .

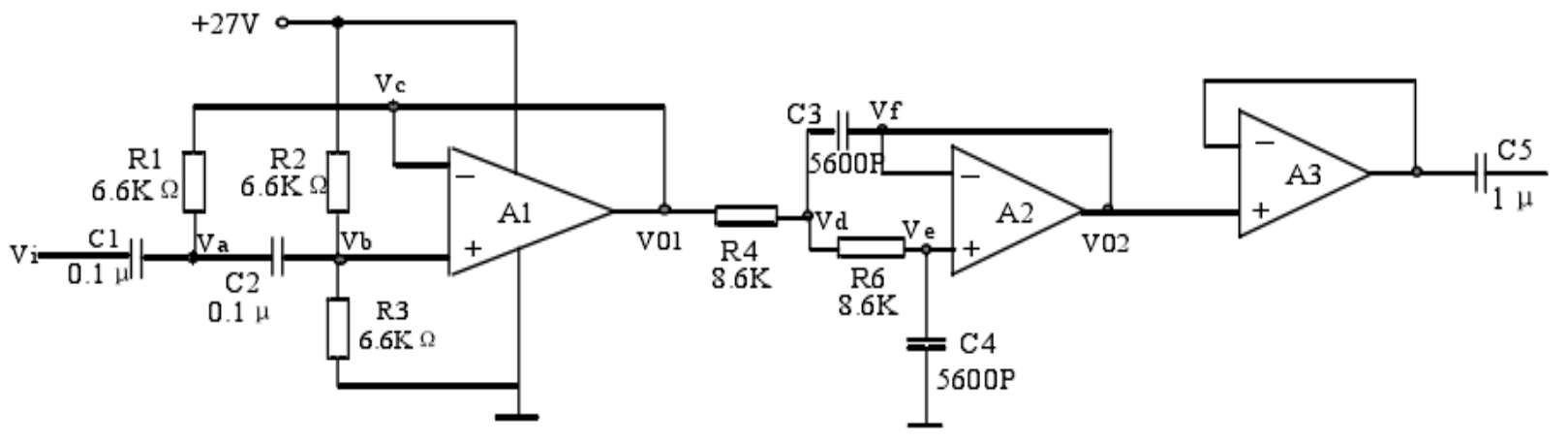

Figure 5 Active band pass filter

As shown in the diagram, R1, R2, R3, C1, C2 and op amp A1 constitute a high pass filter. R4, R5, C3, C4 and op amp A2 constitute a low-pass filter. The low and high pass filter are connected in series to form a band-pass filter circuit. OP AMP (A3) is an amplifier used to isolate the power amplifier and filter circuit. It also plays an important role in matching the input and output loads.

The cutoff frequency for the high frequency filter is $300 \mathrm{HZ}$. The node equations of and can be listed by KCL.

$$
\begin{aligned}
& \frac{V_{i}-V_{a}}{\frac{1}{s C 1}}=\frac{V_{a}-V_{c}}{R 1}+\frac{V_{a}-V_{b}}{\frac{1}{s C 2}} \\
& \frac{V_{a}-V_{b}}{\frac{1}{S C 2}}=\frac{V_{b}}{R 2 / / R 3} \\
& V 01=V_{c}=V_{b} \\
& R 1=R 2=R 3=R=6.6 \mathrm{~K}
\end{aligned}
$$

The simultaneous equations are solved according to formulas (1), (2), (3) and (4). The transfer function of the second order high frequency filter is.

$$
A(S)=\frac{s^{2}}{s^{2}+\frac{1}{R C} s+\frac{1}{\frac{1}{2} R^{2} C^{2}}}
$$

The typical expression of the circuit transfer function of the second order high pass filter is:

$$
A(S)=\frac{A o S^{2}}{s^{2}+\frac{\omega_{n}}{Q} s+\omega_{n}^{2}}
$$

Compared with formula (5) and (6), the equation, $\omega_{N}=\frac{\sqrt{2}}{R C}, Q=\sqrt{2}$ can be obtained.

The cutoff frequency of the high pass filter can be obtained by bringing its value in numerical value. 
The expression of amplitude frequency $S=j_{W}$ in formula (5).

response is obtained by bringing the equation

$$
20 \lg \left|\frac{A(j W)}{A_{0}}\right|=201 g \frac{1}{\sqrt{\left[\left(\frac{\sqrt{2}}{w R C}\right)^{2}-1\right]^{2}+\left(\frac{1}{w R C}\right)^{2}}}
$$

According to formula (7), the amplitude frequency response of the second order high pass filter can be obtained.

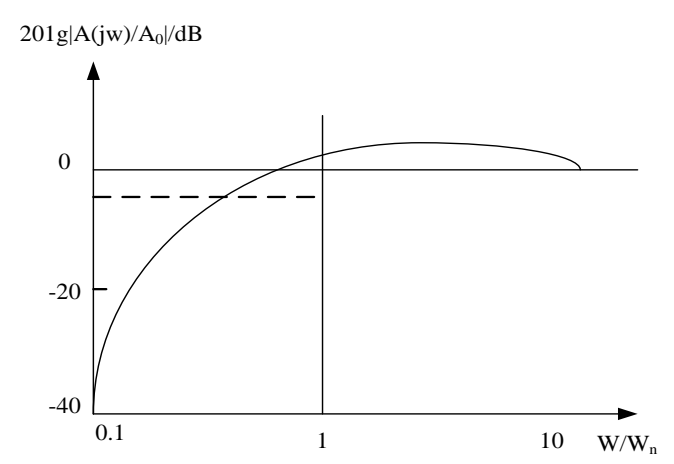

Figure 6 Amplitude frequency response of second order high pass filter

The attenuation rate is $40 \mathrm{~dB} /$ ten octave. For lowpass filters, node equations are also obtained as follows:

$$
\begin{aligned}
& \frac{V 01-V_{\mathrm{d}}}{R 4}=\frac{V_{\mathrm{d}}-V_{e}}{R 6}=\frac{V_{\mathrm{d}}-V_{F}}{\frac{1}{S C 3}} \\
& \frac{V_{\mathrm{d}}-V_{e}}{R 6}=\frac{V_{\mathrm{e}}}{\frac{1}{S C 4}} \\
& \begin{array}{l}
V F=V_{e}=V 02 \\
R 4=R 6=R \\
C 3=C 4=C
\end{array}
\end{aligned}
$$

According to the above, the following equation can be obtained,

$$
A(s)=\frac{\frac{1}{R^{2} C^{2}}}{s^{2}+\frac{2}{R C} s+\frac{1}{R^{2} C^{2}}}
$$

The typical expression of the transfer function of the second order low-pass filter circuit is:

$$
A(s)=\frac{A o \omega_{N}^{2}}{s^{2}+\frac{\omega_{n}}{Q} s+\omega_{n}^{2}}
$$

Compared with formula (12) and (13), the equation, $\omega_{N}=\frac{1}{R C}, Q=1 / 2$ is obtained.

The cutoff frequency of the low-pass filter can be obtained by bringing its value in the numerical value. According to formula (14), the amplitude frequency response of the second order low-pass filter can be obtained.

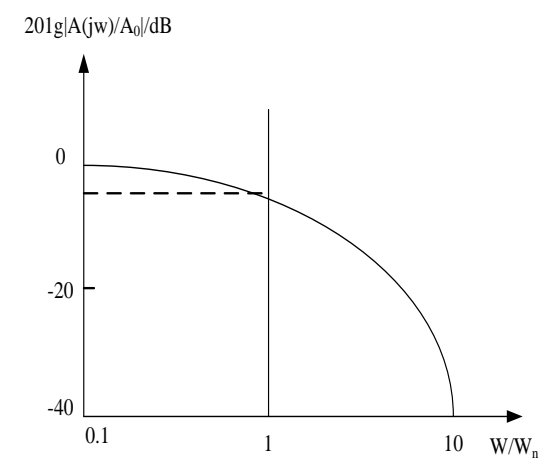

Figure 7 Amplitude frequency response of second order low pass filter

Its attenuation rate is $40 \mathrm{Db} /$ ten octave. The filtering works very well. The high pass and low pass are cascaded to obtain bandpass filters. The amplitude frequency characteristic curve is shown in figure 8, and the audio signal filtered by the bandpass filter is output to the power amplifier.

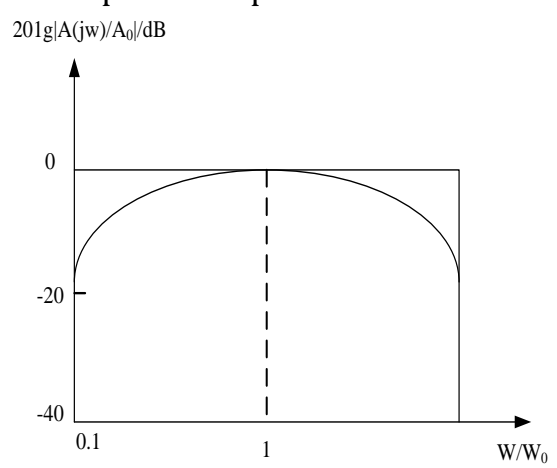

Figure 8 Amplitude frequency characteristic curve 
Power amplifier: It has a wide range of temperatures on the airplane. Generally, when the product temperature is in the range of $-25^{\circ} \mathrm{C}$ to $+85^{\circ} \mathrm{C}$, it cannot meet the temperature requirements of airplane. OPA541 can be used because its temperature range is $-55^{\circ} \mathrm{C}$ to $125^{\circ} \mathrm{C}$. The service voltage of OPA541 is $40 \mathrm{~V}$. However, the plane can only provide +27 volts. In the connection of the circuit, a bridge push-pull power amplifier circuit is adopted, which means that the BTL power amplifier circuit is used to ensure a larger output power at a lower supply voltage.
Output control circuit: The output control circuit consists of transformer, relay, potentiometer and so on. It is used to control the transmit output and the high resistance output. The output voltage of the power amplifier is converted into three voltage values of $1 \mathrm{~V}, 120 \mathrm{~V}$ and $15 \mathrm{~V}$ after transformer conversion. The input of a radio station in which $1 \mathrm{~V}$ voltage is added to transmit voice signals to the ground. The $120 \mathrm{~V}$ voltage is added to the high resistance headphone, and the $15 \mathrm{~V}$ voltage is added to the low resistance headphone.

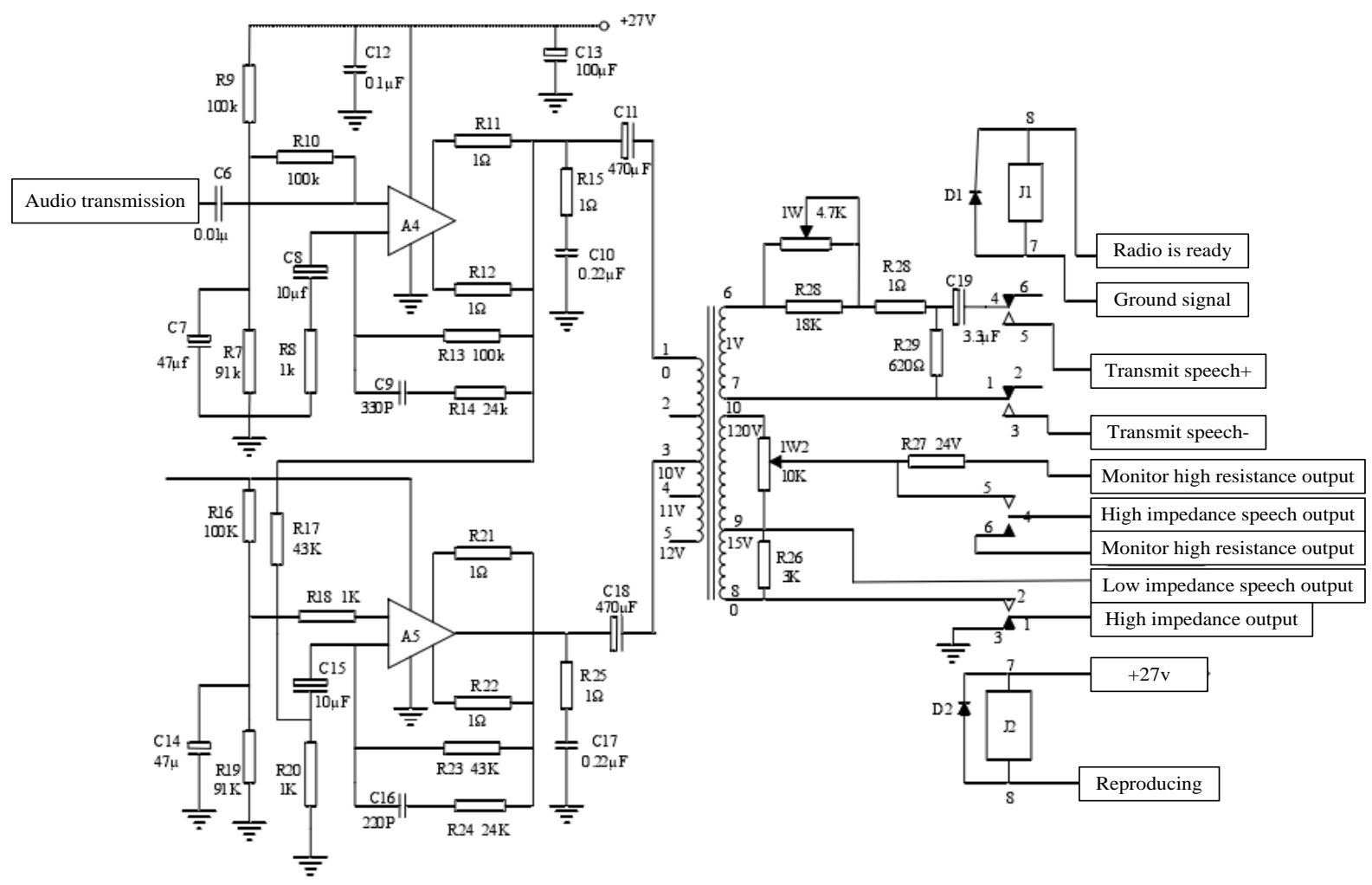

Figure 9 Audio unit power amplifier and output control chart

\subsection{Output unit}

The output unit consists of two output latches, two 7-way integrated drivers, one optocoupler and 14 relays. It is used to output control signals that are interconnected with the aircraft. At the same time, it can also control the work of airborne ultrashort wave radio and voice distribution.

When the sensor signal is read into the microcontroller and processed by software, according to the corresponding storage address of the voice signal in the chip, the corresponding data command is output through the microcontroller to latch the data for the 54LS273. According to the control needs of single chip microcomputer, 54LS273 should drive 14 relays.
Depending on whether the relay is operating or not, the corresponding circuit is connected or disconnected, so that the voice signal can be output along these circuits to the corresponding headphones, microphone or radio.

The driving current of the relay is very large. The output current of the 54LS273 is very small, and it cannot drive the relay directly. Therefore, the circuit uses the ULS2003A driver. ULS2003A is a common Darlington tube array with an output current of $500 \mathrm{~mA}$ and an output voltage of up to 50V. An inductive diode is suppressed inside the integrated chip to eliminate the induction of the load.

The operating voltage is 27 volts and sufficient current is provided to drive the relay. When the corresponding pin is output, the ground level is provided for the relay. It enables the relay to continue working and to connect or disconnect the corresponding voice output circuit. 


\section{Conclusions}

The single-chip microcomputer is used to control the solid-state audio chip through I/O interface for playback, recording and other operations. For the original wire tape, the voice storage time needs to be re-recorded after one year. However, the voice recorded by the semiconductor chip can be stored for 100 years without power consumption.

The aircraft components of the original system need to be characterized by high pressure resistance, heat resistance and stable operation performance. In view of this characteristic, in the overall design process of the circuit, the OPA541 power amplifier and the corresponding circuit components are selected. A circuit with high output efficiency is adopted to reduce the power consumption of the circuit from 100 watts to 35 watts. The improved intelligent voice system has an external dimension of $110 \times 223 \times 186 \mathrm{~mm}$ and its weight is $5.0 \mathrm{~kg}$. In order to ensure the stability of the system, watchdog circuit is used to monitor and reset. Therefore, the improved system fundamentally overcome the defects of the original system of steel tape broadcast mode. It realizes the intelligence, solid and integration of the airborne voice information system.

The main technical indexes and reliability are superior to those of the original system.

\section{References}

[1] Paux, J., Morin, L., Brenner, R., \& Kondo, D. (2015). An approximate yield criterion for porous single crystals. European Journal of Mechanics A/Solids, 51 , 1-10. https://doi.org/10.1016/j.euromechsol.2014.11. 004

[2] Poulios, K., \& Renard, Y. (2015). An unconstrained integral approximation of large sliding frictional contact between deformable solids. Computers \& Structures, 153, 75-90. https://doi.org/10.1016/j.compstruc.2015.02.02 7

[3] Coughlan, L., \& Lambe, T. (2015). Measuring cellular immunity to influenza: methods of detection, applications and challenges. Vaccines,3(2), 293-319. https://doi.org/10.3390/vaccines3020293

[4] Shameer, K., Badgeley, M. A., Miotto, R., Glicksberg, B. S., Morgan, J. W., \& Dudley, J. T. (2017). Translational bioinformatics in the era of real-time biomedical, health care and wellness data streams. Briefings in Bioinformatics,18(1),105.https://doi.org/10.109 3/bib/bbv118

[5] Fatahi, L., Moradi, S., \& Ghanbarzadeh, A. (2015). The application of the geometric offset method to the rigid joint modeling in the differential quadrature element model updating of frame structures. Meccanica,50(6), 1509-1525. https://doi.org/10.1007/s11012-015-0103-6

[6] Mueller, J. B., Zhao, Y. J., \& Garrard, W. L. (2015). Optimal ascent trajectories for stratospheric airships using wind energy. Journal of Guidance Control \& Dynamics, 32(4), 1232-1245. https://doi.org/10.2514/6.2009-1903

[7] Teschke, R., Schwarzenboeck, A., Frenzel, C., Schulze, J., Eickhoff, A., \& Wolff, A. (2016). The mystery of the hawaii liver disease cluster in summer 2013: a pragmatic and clinical approach to solve the problem. Annals of Hepatology, 15(1), 91.

[8] Mettas, C., Agapiou, A., Themistocleous, K., Neocleous, K., Hadjimitsis, D., \& Michaelides, S. (2016). Risk provision using field spectroscopy to identify spectral regions for the detection of defects in flexible pavements. Natural Hazards, 1(1), 1-14. 